\title{
Análise dos impactos na migração do ERP on-premises para cloud computing em uma organização do ramo da construção civil
}

Analysis of impacts on migration from ERP on-premises to cloud computing in an organization in the civil construction industry

Análisis de los impactos en la migración de ERP en las instalaciones a cloud computing en una organización de construcción cicil

Guilherme Augusto Malaquias ORCID: https://orcid.org/0000-0002-1529-4393 Instituto Federal de São Paulo, Brasil

E-mail: guilherme.amalaquias@ outlook.com Letícia Souza Netto Brandi ORCID: https://orcid.org/0000-0002-7473-6008 Instituto Federal de São Paulo, Brasil E-mail: prof.leticia@ifsp.edu.br

\begin{abstract}
Resumo
O presente estudo analisou os impactos de um projeto de migração do sistema ERP On-Premises de uma empresa brasileira de grande porte do ramo da construção civil para servidores em Cloud Computing, analisando as principais modalidades de Cloud Computing disponíveis no mercado brasileiro e qual a melhor alternativa de migração para o cenário atual da empresa. A metodologia de pesquisa deste trabalho tem como base o caráter exploratório e descritivo com o levantamento de informações através de pesquisas bibliográficas de literatura, pesquisas documentais e estudos de caso com coleta de dados através de documentação direta e indireta e análise qualitativa do material com o objetivo de identificar os principais desafios relacionados a implantação de sistemas ERP em cloud computing. Os resultados obtidos com a pesquisa realizada apontaram que o investimento em servidores On-Premises trouxe um melhor resultado e desempenho computacional no momento atual da empresa. Como considerações finais pode-se analisar que a realização de uma POC se mostrou fundamental para expor os diversos problemas que poderiam ocorrer durante o projeto, mostrando-se de suma importância para uma tomada de decisão mais assertiva, onde foi possível verificar que a escolha assertiva de uma metodologia de entrega de Cloud Computing que se alinhe aos resultados esperados pelo negócio é essencial para o sucesso do projeto de migração do ERP.
\end{abstract}

Palavras-chave: ERP (Enterprise Resource Planning); POC (Proof of Concept); SaaS; Cloud computing; Onpremises.

\begin{abstract}
This study analyzed the impacts of a project to migrate the ERP On-Premises system of a large Brazilian company in the construction industry to Cloud Computing servers, analyzing the main Cloud Computing modalities available in the Brazilian market and which is the best migration alternative for the current scenario of the company. The research methodology of this work is based on the exploratory and descriptive character with the collection of information through bibliographic literature research, documentary research and case studies with data collection through direct and indirect documentation and qualitative analysis of the material with the objective to identify the main challenges related to the implementation of ERP systems in cloud computing. The results obtained from the research carried out indicated that the investment in on-premises servers brought better results and computational performance at the company's current moment. As final considerations, it can be analyzed that carrying out a POC proved to be essential to expose the various problems that could occur during the project, proving to be of paramount importance for a more assertive decision-making, where it was possible to verify that the assertive choice A Cloud Computing delivery methodology that aligns with the results expected by the business is essential for the success of the ERP migration project.
\end{abstract}

Keywords: ERP (Enterprise Resource Planning); POC (Proof of Concept); SaaS, Cloud computing; On-premises.

\section{Resumen}

Este estudio analizó los impactos de un proyecto para migrar el sistema ERP On-Premises de una gran empresa brasileña de la industria de la construcción a servidores de Cloud Computing, analizando las principales modalidades de Cloud Computing disponibles en el mercado brasileño y cuál es la mejor alternativa de migración para el escenario 
actual de la empresa. La metodología de investigación de este trabajo se basa en el carácter exploratorio y descriptivo con la recolección de información a través de la búsqueda de literatura bibliográfica, investigación documental y estudios de casos con recolección de datos a través de documentación directa e indirecta y análisis cualitativo del material con el objetivo de identificar los principales desafíos relacionados con la implementación de sistemas ERP en la computación en la nube. Los resultados obtenidos de la encuesta mostraron que la inversión en servidores OnPremises trajo mejores resultados y desempeño computacional en el momento actual de la empresa. Como consideraciones finales, se puede analizar que la realización de un POC resultó fundamental para exponer los distintos problemas que pudieran presentarse durante el proyecto, resultando de suma importancia para una toma de decisiones más asertiva, donde se pudo constatar que la elección asertiva una metodología de entrega de Cloud Computing que se alinee con los resultados esperados por la empresa es esencial para el éxito del proyecto de migración de ERP.

Palabras clave: ERP (Enterprise Resource Planning); POC (Proof of Concept); SaaS; Cloud computing; Onpremises.

\section{Introdução}

Face ao aumento da competição global e o crescimento das expectativas dos consumidores, as empresas estão buscando a excelência operacional, e a implantação de um sistema ERP (Enterprise Resource Planning) tem sido uma opção amplamente adotada (Beheshi, Blaylock, Henderson, Lollar, 2014, 480-501). Mas para que a empresa possa aumentar a sua eficácia operacional com a adoção de um ERP, é importante que essa estratégia esteja alinhada à estratégia e a visão da própria organização (Ekman, Thilenius, Windahl, 2014).

Devido à dificuldade de alguns gestores de TI e dos departamentos financeiros em levantar todos os requisitos para decidir qual tecnologia adotar na implantação do ERPs, muitas vezes geram grandes transtornos e impactos negativos para as empresas. A adoção de sistemas ERP para apoio às atividades de gestão nas empresas industriais atualmente tende a ser um critério qualificador no mercado, visto que cada vez mais o uso de recursos computacionais traz benefícios aos que o utilizam. Porém os custos associados a essa utilização tendem a ser altos e permanentes. Nesse contexto, um novo paradigma computacional denominado cloud computing surge como alternativa para redução desse investimento (Junior, Pires, 2010). A Cloud Computing surge como um novo paradigma para a implantação de aplicações onde os recursos computacionais são fornecidos como um serviço através de uma conexão de rede. Esses serviços são disponibilizados em forma de Software, infraestruturas e plataformas, onde usuários podem adquiri-los sob demanda (Sousa, 2010).

O estudo aborda pontos como o processo de migração de um ambiente On-premises para nuvem, bem como as vantagens, desvantagens e a comparação dos principais métodos de implantação de ERPs em nuvem em relação a implantação de ERPs utilizando recursos de Data Centers internos na própria empresa, como hardwares e softwares - itens que muitas vezes já tem seu valor embarcado nos ERPs na modalidade em nuvem.

Para a realização deste estudo, descreve-se as questões de pesquisa que se fizeram relevantes para a elaboração das pesquisas bibliográficas e do estudo de caso:

- Qual o impacto da migração de um sistema ERP em ambiente On-Premises para cloud computing no desempenho operacional e na estrutura organizacional da área de TI de determinada empresa?

- Quais fatores críticos significativos estão diretamente ligados ao sucesso da implantação do ERP em nuvem em determinada organização?

- Qual o custo x benefício do investimento em tecnologia direcionado ao ambiente On-Premises ou cloud computing em determinada organização?

O estudo de caso foi realizado em uma empresa nacional de grande porte, do ramo da construção civil. A empresa, com sede localizada no estado de São Paulo, conta com cerca de 5 mil colaboradores distribuídos em 12 unidades e diversas frentes de serviço espalhadas pelo país. Apesar da grande quantidade de colaboradores, cerca de $10 \%$ desse total são colaboradores dedicados às áreas administrativas, estratégicas e táticas. O restante - e grande maioria dos colaboradores - são de áreas operacionais, trabalhadores de campo, exercendo atividades da área da construção civil pesada. Por possuir uma 
operação com administrativo enxuto, controlando diversas unidades de serviço espalhadas pelo Brasil, um controle rígido de produção se faz essencial para garantir a visibilidade e a viabilidade da operação. A empresa, que denominaremos no artigo como "Empresa X" além do ramo da construção civil, faz parte de um grupo de outras empresas das áreas de mineração, indústria, tecelagem e agropecuária.

Devido a oportunidade de expansão dos negócios e a necessidade do aumento do controle de produção se fez de extrema importância e necessidade a adoção de uma ferramenta que fosse capaz de medir o avanço físico da produção e de viabilizar uma análise de desempenho do nível operacional e gerencial. Em paralelo, o ambiente computacional e de servidores da organização, que era On-Premises, ou seja, adquiridos e alocados dentro da própria empresa, foram adquiridos no ano de 2014 e já estavam no final de seu ciclo de vida. Isto posto, cabendo assim, aos gerentes de TI e ao nível estratégico alinharem as estratégias de TI com as estratégias da organização, visando decidir entre a atualização do parque de servidores e equipamentos, ou a migração dos sistemas ERP para servidores em nuvem, buscando uma maior elasticidade e escalabilidade em termos de desempenho para atender ao novo projeto do sistema de gestão do avanço físico das obras.

Diante do exposto, o presente artigo tem como objetivo geral analisar os impactos na migração do sistema ERP local para nuvem portanto estabelece os seguintes objetivos específicos:

- Analisar o impacto da migração de um sistema ERP implementado em um ambiente On-premises para cloud computing no desempenho operacional e na estrutura organizacional de TI de determinada empresa;

- Verificar os principais fatores que podem contribuir negativamente para a execução do projeto de migração do ERP;

- Levantar as principais modalidades de cloud computing existentes no mercado atual, adequando a necessidade do escopo do projeto em determinada empresa;

- Identificar as principais vantagens e desvantagens em se manter um ambiente On-premises internamente na empresa ou migrar o ambiente para cloud computing;

\section{Metodologia}

A metodologia de pesquisa deste trabalho tem como base o caráter exploratório e descritivo com o levantamento de informações através de pesquisas bibliográficas de literatura, pesquisas documentais e estudos de caso com coleta de dados através de documentação direta e indireta e análise qualitativa do material com o objetivo de identificar os principais desafios relacionados a implantação de sistemas ERP em cloud computing.

Segundo Triviños (1987, p. 175) o estudo de caso é uma categoria de pesquisa qualitativa cujo objeto é uma unidade que se analisa profundamente, tendo como objetivo aprofundar a descrição de determinada realidade. Triviños (1987, p. 175) ainda defende que o grande valor do estudo de caso é fornecer o conhecimento aprofundado de uma realidade delimitada que os resultados atingidos podem permitir e formular hipóteses para o encaminhamento de outras pesquisas.

A pesquisa qualitativa não procura enumerar e/ou medir os eventos estudados, nem emprega instrumental estatístico na análise dos dados, envolve a obtenção de dados descritivos sobre pessoas, lugares e processos interativos pelo contato direto do pesquisador com a situação estudada, procurando compreender os fenômenos segundo a perspectiva dos sujeitos, ou seja, dos participantes da situação em estudo (Godoy, 1995, p58).

Segundo Gil (1991, p. 46) um trabalho é de natureza exploratória quando envolver levantamento bibliográfico, entrevistas com pessoas que tiveram (ou tem) experiências práticas com o problema pesquisado e análise de exemplos que estimulem a compreensão. Possui ainda a finalidade básica de desenvolver, esclarecer e modificar conceitos e ideias para a formulação de abordagens posteriores. Dessa forma, este tipo de estudo visa proporcionar um maior conhecimento para o pesquisador acerca do assunto, a fim de que esse possa formular problemas mais precisos ou criar hipóteses que possam ser pesquisadas por estudos posteriores (Gil, 1999, p. 43). 


\section{Referencial teórico}

\subsection{A evolução dos sistemas ERP e da infraestrutura de servidores}

Os sistemas ERP (Enterprise Resource Planning ou Sistema de Gestão Integrados) surgiram em meados da década de 70, com a evolução do MRP (Materials Requirements Planning ou Sistema de Planejamento de Necessidades Materiais). O ERP tem como um de seus objetivos, auxiliar os gestores de empresas a terem facilmente uma visão gerencial de seus negócios e de seus processos internos, bem como a integração de atividades de diferentes setores, como vendas, finanças, compras, contabilidade, recursos humanos, entre outros. Visando a centralização das informações em uma única plataforma, com a utilização de sistemas ERP o fluxo de dados corporativo se torna mais fluído e ao mesmo tempo elimina a duplicidade de informações e a burocracia dos controles e processos administrativos.

Com o passar dos anos, o aumento do número de empresas e consequentemente o aumento da concorrência dentro de um mesmo setor, cada vez mais se faz necessário que as empresas busquem vantagens competitivas perante seus concorrentes, sempre se utilizando de novas tecnologias que surgem no mercado com o intuito de reduzir falhas humanas, automatizar e agilizar processos manuais e garantir a integridade, confidencialidade e disponibilidade de seus dados. Para que a implantação dessas ferramentas pudesse ser viabilizada, o investimento em infraestrutura de telecomunicação, redes e servidores que comportasse e garantisse o bom desempenho e disponibilidade das aplicações se fez necessário em paralelo ao investimento em ferramentas e softwares.

Com a maior integração e informatização dos processos empresariais e a conversão de dados de meios físicos (folhas de papel, ofícios etc.) para meios digitais (planilhas, bases de dados etc.) bem como a necessidade de formas eficientes de extração e interpretação destes dados em informações para o negócio, o aumento da dependência das empresas perante o uso de softwares foi inevitável. Para que isso pudesse ser entregue de forma eficiente para todos os setores e colaboradores das organizações, surgiu o conceito de Data Center tradicional, ou Data Center On-Premises. Um data center tradicional é formado por uma grande estrutura de servidores físicos alocados dentro da própria empresa com o intuito de hospedar seus dados e serviços em um único local para tê-los sempre à disposição.

Com o passar dos anos cada vez mais essa quantidade de dados e informações vem aumentando, acarretando a necessidade de investimento em servidores que garantam um bom desempenho em poder computacional, porém a longa data, percebeu-se que para muitas organizações esse investimento em ativos físicos se tornou inviável pois diversos fatores geram o alto custo de um Data Center On-Premises, custos estes muitas vezes não calculados a longo prazo, o que pode inviabilizar um projeto de Data Center On-Premises.

Alguns exemplos de custos que devem ser levados em consideração a longo prazo em um projeto de Data Center OnPremises:

- Cálculo do consumo mensal de energia elétrica do Data Center;

- Ter um espaço dedicado, estratégico e seguro onde o Data Center será montado;

- Investimento em refrigeração dos equipamentos do Data Center, o que eleva também o consumo de energia elétrica;

- Investimento em sistemas de segurança da camada física como sistemas anti-incêndio, sistemas de monitoramento, controle de acesso etc.;

- Investimento em sistemas de continuidade do negócio em caso de falhas elétricas, bem como Nobreaks de alta capacidade e geradores de energia;

- Levar em consideração o tempo de depreciação dos equipamentos de rede e servidores, visando um novo investimento ao fim da vida útil dos equipamentos;

- Levar em consideração a aquisição de licenças de Softwares e Sistemas Operacionais bem como o ciclo de vida útil desses sistemas, visando um novo investimento ao fim desse ciclo; 
- Investimento em equipe técnica capacitada para a configuração e gerenciamento desses equipamentos, entre outros.

Estes são alguns dos diversos exemplos de custos que muitas vezes não são levados em consideração e que podem acabar inviabilizando o projeto de Data Center On-Premises, ou pior, causando um grande impacto financeiro dependendo do tamanho da operação da empresa, da criticidade dessa operação e da área de atuação da organização.

A fim de reduzir esse investimento na aquisição, implantação e manutenção de Data Centers On-Premises e visando que as organizações foquem no que realmente traz valor agregado ao negócio, como seu Core Business e a expansão de seus negócios, surgiu o conceito de Data Centers em nuvem - ou Cloud Computing, porém adaptar essa modalidade de computação em uma organização que já possui uma infraestrutura de TI baseada em Data Center On-Premises bem definida e que supre as necessidades de negócio, pode ser tornar um desafio a se superar. O processo de migração de um ERP que já se encontra em produção, pode gerar um grande impacto operacional, para isso é imprescindível que um bom planejamento de migração seja realizado e principalmente a verificação se a aplicação entrega o desempenho esperado no ambiente de Cloud Computing, para isso, diversos testes e homologações devem ser realizados.

Um dos métodos utilizados para a homologação de um sistema ou funcionalidade é a realização de uma POC (Proof of Concept ou Prova de Conceito). Segundo Carsten (1989) "Uma prova de conceito serve para denominar um circuito construído seguindo métodos similares aos de um protótipo convencional, no qual, entretanto a intenção é unicamente demonstrar que um determinado circuito ou uma nova técnica de produção é factível”. A PoC é importante porque verifica a viabilidade do projeto, complexidade, custos, mercado e o tempo necessário de execução de todas as etapas. Dessa forma, ela é essencial para a empresa como um todo, uma vez que traz segurança para o investimento (Website Gaea, 2021).

\subsection{Paradigma da computação em nuvem}

A computação em nuvem (Cloud Computing) é um paradigma que consiste em combinar infraestruturas virtuais, escaláveis e com gestão automática de recursos, partilhadas no mesmo modelo de negócio. A forma de definir os custos, designada por pay as you go (em português, pague pelo que usar), é baseada no uso. Procurando garantir uma constante adaptação às exigências do negócio, a computação em nuvem proporciona confiança e qualidade de serviço, reduzindo o risco associado ao lançamento de aplicações e o tempo de resposta (Gomes, 2012).

Segundo Teles (2020) A computação em nuvem é um paradigma que permite o acesso à rede a um pool escalável e elástico de recursos físicos ou virtuais compartilháveis com provisionamento de autoatendimento e administração sob demanda.

Na visão do cliente que visa aderir a Cloud Computing, a ideia é contratar um serviço, por tempo determinado ou não, que disponibilizará a infraestrutura de TI necessária para seu negócio ou somente um serviço para que suas aplicações possam ser publicadas e disponibilizadas para seus colaboradores ou seus clientes. Dessa forma, é possível fazer a contratação de serviços de TI, pagando em formato de assinatura, ao invés um grande investimento em equipamentos que se tornarão obsoletos e precisarão ser reparados e trocados com o tempo. Assim é possível realizar a troca do CAPEX ${ }^{1}$ por OPEX ${ }^{2}$, otimizando os custos com infraestrutura de TI e pagando somente pelo que for utilizado. Os serviços de Cloud Computing podem ser disponibilizados em diversas modalidades, porém explicaremos a seguir as principais modalidades disponibilizadas no mercado atual, suas principais aplicações e suas vantagens e desvantagens perante as outras modalidades.

\footnotetext{
${ }^{1}$ Capital Expenditures -Termo utilizado para se referir às despesas de capital realizadas por uma empresa, como o investimento de bens realizados.

${ }^{2}$ Operational Expenditure - Termo utilizado para indicar os custos operacionais realizados por uma empresa, como alocação de serviços, aluguéis de bens etc.
} 


\subsubsection{SaaS (Software as a Service)}

Segundo Augusto (2019), a modalidade SaaS (Software as a Service ou Software como um serviço) é uma das modalidades de fornecimento de serviços de Cloud Computing mais conhecidas e utilizadas atualmente. Essa modalidade permite aos contratantes usufruir de serviços e programas de forma online e muitas vezes por assinatura, sem a necessidade de instalação e armazenamento em servidores locais ou até mesmo nas estações de trabalho.

Por meio da utilização de Softwares baseados em uma rede online, as empresas podem atingir uma maior escalabilidade de implantação do software apenas pelo acesso à internet. Essa facilidade de implantação aliado ao baixo custo, já que na maioria das vezes é pago mensalmente através assinatura e podendo ser cancelado a qualquer momento, sem multas (dependendo do contrato realizado), fornece infraestrutura de forma externa e confiável.

As principais características do modelo SaaS são:

- Acesso a aplicações, serviços e dados via Web ou aplicativos móveis;

- Gerenciamento centralizado de licenças, usuários e permissões;

- Possibilidade de realizar integrações e personalização das ferramentas por meio de APIs (Application Programming Interfaces).

Alguns exemplos de aplicações SaaS muito difundidas e utilizadas por diversas organizações são ferramentas de produtividade como, Microsoft Office 365, Google Gsuite, ferramentas de Webmail, chats, videoconferência, plataformas de CRM, entre outros.

\subsubsection{PaaS (Platform as a Service)}

Segundo Augusto (2019), a modalidade PaaS (Platform as a Service ou Plataforma como um serviço) é semelhante ao SaaS. A diferença entre as duas modalidades é que ao contrário do SaaS que se refere a um Software entregue pela Web, o PaaS trata-se de um ambiente ou uma plataforma, para criar, hospedar e gerir um Software próprio, onde o usuário pode instalar e gerenciar suas próprias aplicações ou de terceiros, utilizando as ferramentas e bibliotecas oferecidas pelo provedor. Esse é um modelo muito utilizado em empresas que desenvolvem softwares internamente. Esse modelo, além de permitir a criação de aplicações, realizada em nuvem, possibilita que a empresa contratante consiga testar, analisar, gerenciar e executar todas as suas ações.

As principais características do modelo PaaS são:

- Serviços já vem pré-programados de fábrica;

- Permite o gerenciamento e acesso a um grande volume de dados extensos e complexos;

- Permite atualização e acesso aos aplicativos hospedados na rede via dispositivos móveis;

- Redução do tempo de codificação já que possui componentes pré-codificados;

- Alta flexibilidade pois têm suporte a diversas plataformas.

- Possui funcionalidades para o ciclo de vida completo de aplicações web, desde testes até atualizações de produto.

Alguns exemplos de plataformas como um serviço muito utilizados são:

- Google Cloud: Java, Python, PGP e Go;

- Microsoft Azure: .NET, Node.js; PHP, Python, Java e Ruby;

- Amazon Web Services (AWS): Java, .NET, PHP, Node.js, Python, Ruby, Docker;

- Heroku: Ruby, Python, Java, Scala, Clojure e Node.js. 


\subsubsection{IaaS (Infraestucture as a Service)}

Segundo o Augusto (2019), na modalidade IaaS (Infraestructure as a Service ou infraestrutura como um serviço) as empresas adquirem o volume de recursos de hardware que precisam em formato de assinatura. Toda a disponibilização de recursos de hardware e sistemas operacionais é entregue pelo provedor ao contratante através de um Data Center em nuvem. A empresa, por sua vez, faz a configuração dos servidores e serviços como bem entender, entregando as aplicações para os clientes de sua rede interna. O modelo IaaS, atualmente, pode ser dividido em quatro subcategorias para adoção das empresas:

- Nuvem Pública: São ambientes em nuvem, geralmente criados em uma Infraestrutura de TI que não é de propriedade da empresa contratante, sendo fornecidos pelos provedores de Cloud. As nuvens públicas tradicionais eram executadas em ambientes off-premises (fora de casa). Atualmente, provedores de serviços em nuvem oferecem serviços nos data centers on-premises dos clientes. Todas as clouds se tornam públicas quando os ambientes são particionados e redistribuídos para vários locatários (Website RedHat, 2021).

- Nuvem Privada: Segundo o RedHat (2021), nuvens privadas são definidas como ambientes de nuvem dedicados a um usuário final. $\mathrm{O}$ ambiente geralmente é executado por trás do firewall desse usuário. Todas as nuvens se tornam privadas quando a infraestrutura de TI subjacente é dedicada e o cliente tem acesso totalmente isolado a ela. As nuvens privadas não precisam mais ser baseadas em infraestrutura de TI on-premises. Atualmente, algumas organizações estão criando nuvens privadas em data centers alugados localizados off-premises. As nuvens privadas podem ser divididas em dois subtipos:

- Nuvens Privadas Gerenciadas: Os clientes criam e usam uma nuvem privada implantada, configurada e gerenciada por um fornecedor terceirizado. Esse tipo de nuvem são uma opção para empresas com poucos funcionários ou com equipes de TI sem a qualificação necessária para fornecer infraestrutura e serviços de nuvem privada adequados.

- Nuvens Privadas Dedicadas: O conceito é basicamente uma nuvem dentro de outra nuvem. É possível ter uma nuvem dedicada em uma nuvem pública ou em uma nuvem privada. Por exemplo, é possível implantar uma nuvem dedicada para um determinado departamento dentro da nuvem privada da própria organização.

- Nuvens Híbridas: Segundo o RedHat (2021), nuvens híbridas caracterizam-se por um ambiente de TI aparentemente único, criado a partir de vários outros ambientes conectados. As nuvens híbridas podem incluir:

- No mínimo, uma nuvem privada e uma nuvem pública;

- Duas ou mais nuvens privadas;

- Duas ou mais nuvens públicas;

- Um ambiente virtual ou físico conectado a, no mínimo, uma nuvem pública ou privada.

- Multicloud: A multicloud é um modelo de cloud composto por mais de um serviço e de um provedor de cloud, sendo ela pública ou privada. Um ambiente multicloud pode existir propositalmente (para uma melhor redundância de dados, recuperação de desastres ou redução de custos) ou involuntariamente (como resultado de TI invisível). De uma forma ou de outra, ter várias nuvens está se tornando mais comum entre empresas que buscam melhorar a segurança e o desempenho em uma gama maior de ambientes (Website RedHat, 2021). 
A Figura 1 mostra a separação de responsabilidades por camada das diversas modalidades de Cloud Computing em relação a infraestrutura On-Premises.

Figura 1 - Separação da Responsabilidade para Cloud Computing.

\begin{tabular}{|c|c|c|c|}
\hline $\begin{array}{l}\text { TI Tradicional } \\
\text { (On premises) }\end{array}$ & $\begin{array}{l}\text { laaS - Infraestrutura } \\
\text { como Serviço }\end{array}$ & $\begin{array}{l}\text { PaaS - Plataforma } \\
\text { como Serviço }\end{array}$ & $\begin{array}{l}\text { SaaS - Software } \\
\text { como Serviço }\end{array}$ \\
\hline Aplicações & Aplicações & Aplicações & Aplicações \\
\hline Dados & Dados & Dados & Dados \\
\hline Runtime & Runtime & Runtime & Runtime \\
\hline Middleware & Middleware & Middleware & Middleware \\
\hline $\mathrm{O} / \mathrm{s}$ & $\mathrm{O} / \mathrm{s}$ & $\mathrm{O} / \mathrm{s}$ & $\mathrm{O} / \mathrm{s}$ \\
\hline Virtualização & Virtualização & Virtualização & Virtualização \\
\hline Servidores & Servidores & Servidores & Servidores \\
\hline Storage & Storage & Storage & Storage \\
\hline Redes & Redes & Redes & Redes \\
\hline
\end{tabular}

Responsabilidade sob a Segurança

Fonte: Jornada para nuvem (2021).

\subsection{Estudo de caso}

Conforme mencionado na introdução deste artigo, o estudo de caso foi realizado em uma empresa de grande porte do ramo da construção civil, situada no estado de São Paulo no Brasil e que possui por característica cerca de $90 \%$ de seu quadro de colaboradores dedicados a área operacional e de construção civil, possuindo assim, um quadro enxuto de colaboradores administrativos para gerenciar o operacional da organização.

A empresa atualmente possui todo seu ERP em servidores On-premises com mais de 5 anos de uso, chegando ao fim do ciclo de vida estipulado pelo fabricante, perdendo assim sua garantia e suporte do fabricante. Um outro problema atualmente enfrentado pela empresa são diversas queixas dos usuários referentes a lentidão na utilização do sistema ERP. A fabricante do ERP disponibiliza mensalmente pacotes de atualizações que trazem correções de erros e melhorias de segurança, porém também são implementadas novas funcionalidades ao ERP, exigindo um maior poder computacional ao longo dos anos para que o desempenho do sistema se mantenha em níveis aceitáveis pelo negócio. Além disso, um novo módulo de gestão de obras foi adquirido pela empresa e adicionado ao ERP. Este módulo além de fazer toda a gestão de obras da empresa, executa o cálculo de orçamento de toda a execução da obra, desde os materiais utilizados, até a mão-de-obra especializada e a locação de máquinas e equipamentos. O módulo de gestão de obras se tornou um aliado de extrema importância competitiva para a empresa frente a seus concorrentes, porém exige uma grande capacidade computacional para que execute os cálculos de forma satisfatória.

Visando a renovação de seu ambiente de servidores e a fim de obter uma melhor capacidade computacional para 
atender as novas demandas do negócio, alinhando a estratégia de negócio aos três pilares básicos da segurança da informação Integridade, disponibilidade e confidencialidade dos dados - os gestores de TI da empresa se viram na necessidade de realizar um investimento no ambiente de servidores.

A fim de direcionar o investimento em um modelo de entrega de infraestrutura mais assertivo, que realmente trouxesse o resultado sob o investimento esperado pelo negócio, dois modelos foram avaliados. O primeiro modelo seria a aquisição de novos servidores físicos para a atualização do ambiente On-premises e melhoria de desempenho. O segundo modelo seria a migração do ERP da empresa para um serviço de Cloud Computing disponibilizado pela própria fabricante do ERP, fornecidos através da modalidade SaaS, como já explicados anteriormente. A seguir, serão apresentados os estudos realizados que serviram como base para a tomada de decisão da aplicação do investimento disponibilizado pelo negócio.

\subsubsection{Estudo para a aquisição de novos servidores}

Para o estudo de aquisição de novos servidores, levou-se em consideração a extensão da garantia atual dos equipamentos já adquiridos - estendendo sua vida útil- e a aquisição de um novo servidor para a melhoria de desempenho computacional.

No que se refere a capacidade computacional, a empresa atualmente conta com um conjunto de três servidores físicos, interligados através de balanceamento de carga, garantindo uma alta disponibilidade dos dados e dos sistemas. Estes servidores físicos fazem parte de uma mesma SAN ${ }^{3}$ (Storage Area Network) e seus dados são salvos em um Storage ${ }^{4}$ que atualmente se encontra em sua capacidade máxima de armazenamento.

Devido a grande quantidade de queixas gerada pelos colaboradores referente a lentidão na execução de processos no sistema ERP, um estudo do ambiente de servidores foi realizado pelos desenvolvedores do ERP, a fabricante dos servidores adquiridos pela empresa $\mathrm{X}$ junto a equipe de infraestrutura da empresa $\mathrm{X}$. Na primeira análise, o banco de dados do sistema ERP foi enviado para a empresa desenvolvedora do sistema e testes foram realizados calculando o tempo de execução de um determinado projeto de obra na infraestrutura de servidores da empresa desenvolvedora do sistema, em comparação aos tempos obtidos executando o mesmo projeto na estrutura de servidores da empresa X. Para os testes, foram realizadas 3 execuções do processo de cálculo de projeto e os resultados apresentados na Figura 2 foram obtidos em cada ambiente:

\footnotetext{
${ }^{3}$ SAN (Storage Area Network ou Rede de Área de Armazenamento) - é um tipo de rede privativa de armazenamento dedicada e de alta velocidade voltada para conectar e manter disponíveis servidores e Storages. (Website Synnex, 2021).

${ }^{4}$ Storage - É um equipamento ou Hardware que contém vários discos rígidos interligados e ligados aos servidores através de uma SAN. Seu intuito, além de aumentar a capacidade de armazenamento, é garantir a disponibilidade dos dados armazenados. (Website Infobusiness, 2011).
} 
Figura 2 - Comparação do Tempo de Cálculo de Projeto Entre as Infraestruturas da Empresa X e da Desenvolvedora do ERP.

Tempo de Execução do Processo de Cálculo de Projeto (s)

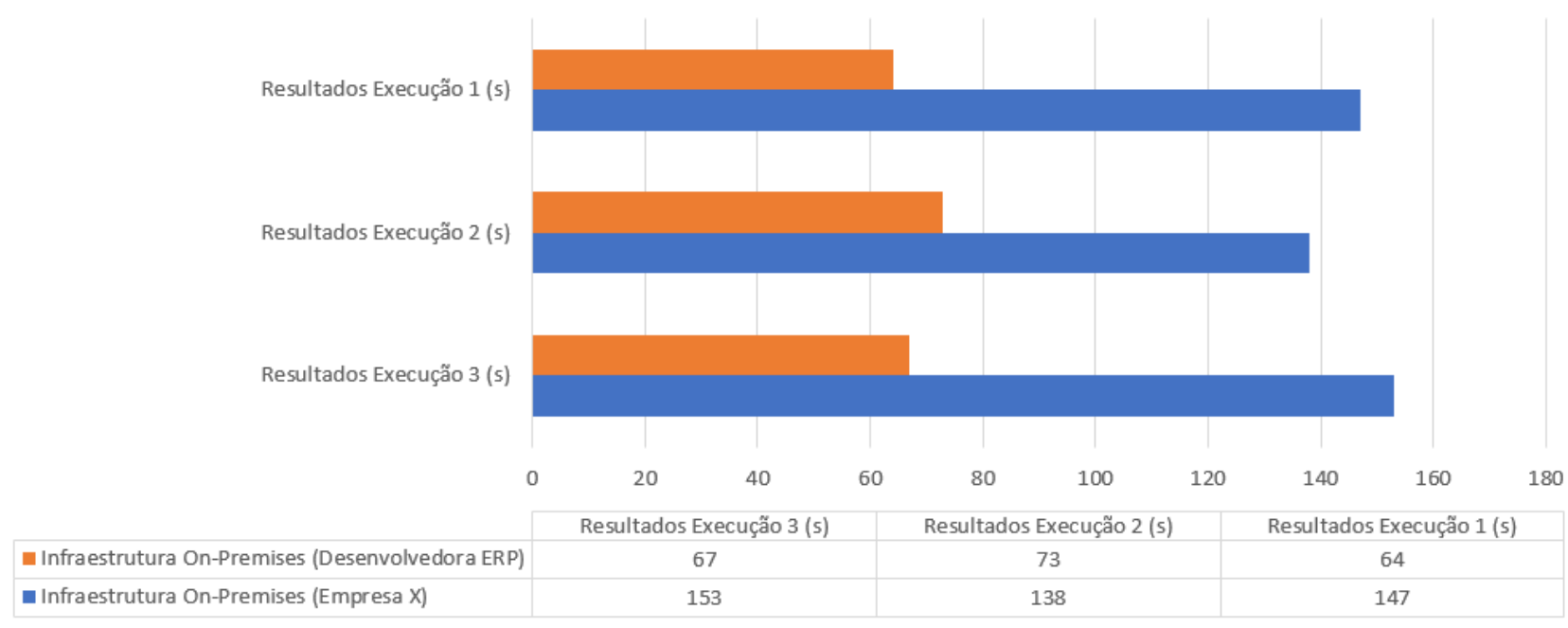

Fonte: Autores (2021).

Após os resultados dos testes realizados na primeira análise, notou-se uma grande diferença nos tempos de execução do cálculo de um mesmo projeto, sendo em média 53,43\% menor, quando executado no ambiente de infraestrutura da desenvolvedora do ERP. Após os resultados obtidos na primeira etapa da análise, uma segunda análise foi realizada, desta vez na infraestrutura de servidores On-premises da empresa X. Notou-se que ao realizar o processo de cálculo do orçamento de obra no sistema ERP, a memória RAM dos servidores chegava a 100\% de uso, mesmo com a CPU sendo utilizada somente em torno de 50\%. Notou-se também que o Storage onde os arquivos do sistema ERP estavam armazenados era composto por discos rígidos que estavam fora da especificação do sistema na versão atual, o que gerava a lentidão no momento da execução de rotinas onde se fazia necessário maior poder computacional.

Após as análises realizadas e a conclusão de que seria necessário o investimento em infraestrutura de servidores, a equipe de infraestrutura da empresa $\mathrm{X}$ realizou o levantamento dos seguintes componentes para que fosse possível a atualização do ambiente computacional e consequentemente a melhoria do desempenho entregue pelo sistema ERP aos colaboradores:

- Aquisição de um novo servidor: Após resultado da análise realizada, contatou-se que seria necessário a aquisição de um novo servidor para ser adicionado ao conjunto atual de servidores, já que todos os três servidores atuais não possuíam mais capacidade de expansão de memória RAM. A soma de um novo servidor aumentaria significativamente a capacidade computacional do ambiente.

- Aquisição de um novo Storage: Para atender os requisitos recomendáveis estipulados pela desenvolvedora do sistema ERP, seria necessário a aquisição de um novo Storage com tecnologia de armazenamento 100\% em SSD para uma melhor entrega do sistema ERP em ambiente de produção. Em busca de uma melhoria em todo o ambiente de servidores virtuais e de outros sistemas complementares além do ERP, todo o ambiente atual de servidores seria migrado para o Storage SSD. O Storage atual com tecnologia de disco rígido seria utilizado na criação de um ambiente de homologação para os sistemas e aplicações da Empresa X. 
- Atualização do Sistema Hypervisor5: Após o término do suporte técnico, a defasagem de versão do Hypervisor atual e a necessidade de realizar a aquisição do sistema para o novo servidor, contatou-se que seria necessário a aquisição da versão atual do sistema Hypervisor para todos os servidores - sendo o servidor novo e os três servidores legados - a fim de garantir a integridade e compatibilidade entre as versões do sistema, minimizando os risco de falhas no sistema Hypervisor em ambiente de produção.

- Aquisição da Licença de Sistema Operacional: Para atender os requisitos de Compliance do licenciamento de Software da Microsoft, seria necessário a aquisição de uma nova licença do Sistema Operacional Windows Server 2016 Datacenter para ser utilizado nas máquinas virtuais que fossem executadas no novo servidor do conjunto. Como o Sistema Operacional dos três servidores legados foi adquirido junto com os servidores, se tratava de uma versão anterior do Sistema Operacional - Windows Server 2012 R2 Datacenter. Com o intuito de reduzir o custo do projeto, e como o Sistema Operacional dos servidores legados encontrava-se dentro do ciclo de vida da Microsoft - recebendo atualizações de segurança e melhorias de desempenho mensalmente - estudou-se a aquisição de somente uma licença do Windows Server 2016 Datacenter para ser colocada no novo servidor e dessa forma seria possível o Downgrade de versão do Sistema Operacional para até duas versões anteriores, dessa forma seria possível a equivalência de versões entre o novo servidor e os servidores legados, assim atendendo o Compliance de Licenciamento da fabricante.

- Atualização do Sistema Gerenciador de Banco de Dados: Com o fim do ciclo de vida do Sistema Gerenciador de Banco de Dados da Microsoft - SQL Server 2008 R2 Datacenter em setembro de 2019 (Microsoft.com - Lifecycle), o sistema passou a não receber atualizações de segurança e de melhorias de desempenho, não sendo mais suportado pelo desenvolvedor do ERP implantado na Empresa X, o que também impediria novas atualizações do Sistema ERP. Dessa forma, seria necessário a aquisição de uma nova licença do SQL Server 2016 Datacenter e posterior migração de versão das bases de dados atuais para compatibilidade com a versão 2016 do SQLServer.

- Renovação de Garantias dos Servidores Legados: A fim de estender o ciclo de vida dos servidores legados, estudou-se estender a garantia do fabricante para esses equipamentos. O prazo máximo de extensão da garantia seria por mais 2 anos, segundo proposta do fabricante.

- Aquisição de um Sistema de Backup: Para suprir a necessidade de backup dos servidores atuais da empresa, um novo sistema de Backup de máquinas virtuais seria necessário e poderia ser utilizado junto ao Storage antigo da empresa, estendendo a utilização deste equipamento.

Após o levantamento do investimento necessário para a atualização do ambiente interno de servidores de forma que atenda as especificações do ERP, também estendendo a vida útil dos servidores atuais, notou-se que além do investimento inicial ser maior do que o esperado pelo nível estratégico da Empresa $X$, seria necessário a contratação de serviços especializados que pudessem fazer a instalação do novo servidor no conjunto de servidores, atualização do sistema Hypervisor, migração das máquinas virtuais para o novo Storage SSD e atualização do banco de dados da empresa para a versão atual do SQLServer, o que demandaria um maior investimento de recursos, um maior tempo para a atualização do ambiente como um todo, podendo gerar um grande impacto na produção da Empresa X, já que todo o processo seria realizado com o ambiente de

${ }^{5}$ Hypervisor - É um processo que cria e executa máquinas virtuais. Um Hypervisor permite que um computador host ofereça suporte a várias máquinas virtuais, compartilhando virtualmente seus recursos como memória e processamento. (Website Vmware, 2021) 
produção atual em operação.

\subsubsection{Estudo para a migração de ERP para ambiente cloud computing}

A fim de estudar novas estratégias que trouxessem bons resultados na solução do problema de desempenho do sistema ERP atual, tivessem um investimento inicial menor - em comparação ao estudo de aquisição de novos servidores - e gerassem pouco impacto na migração do sistema, a Empresa X iniciou o estudo para a migração do sistema ERP atual para Cloud Computing, junto com a empresa desenvolvedora do ERP.

Para a realização do estudo, uma POC foi realizada para a validação do desempenho do sistema adotando a modalidade SaaS de Cloud Computing. Para que a POC fosse realizada em um ambiente mais equivalente possível ao cenário atual da Empresa X, onde seria possível a validação da maior parte das regras do negócio e também onde seriam apontados os possíveis problemas que surgiriam na migração do ERP para SaaS, o Upload de uma cópia da base de dados atual da Empresa $\mathrm{X}$ foi enviado para que a desenvolvedora do ERP pudesse montar o ambiente SaaS de forma mais fiel possível ao atual ambiente On-premises da Empresa X. Após o Upload da base de dados para o ambiente Cloud e os ajustes realizados por parte da desenvolvedora, o ambiente SaaS foi disponibilizado para acesso da Empresa X.

Para a realização dos testes, 50 usuários de variados setores da Empresa $\mathrm{X}$ foram selecionados para a utilização do sistema ERP em SaaS durante um período de 4 semanas. Após a seleção dos usuários, um cliente de comunicação do sistema ERP foi instalado nos computadores para que o sistema pudesse se comunicar com a base de dados em nuvem. Ao fim do período de testes com a aplicação ERP em SaaS, foi possível a constatação dos seguintes problemas:

- Falta de Funcionalidades no Sistema: Por se tratar de um sistema ERP disponibilizado e consolidado a muitos anos no mercado brasileiro, frequentemente passa por atualizações para melhorias de desempenho e tecnologias. Uma das atualizações recentemente realizadas foi a troca da linguagem de programação em que o sistema foi desenvolvido. Para isso, muitas funcionalidades da versão antiga do sistema foram migradas para a nova versão, porém várias funcionalidades ainda estão em processo de migração pela empresa desenvolvedora do ERP e para que os colaboradores tenham acesso as funcionalidades da versão antiga do sistema, é necessário a instalação de uma versão completa do sistema no computador do colaborador. Para acesso ao sistema ERP no formato SaaS, um cliente de comunicação é instalado no computador do colaborador, porém, através desse cliente só é possível acesso as funcionalidades da nova versão do sistema, o que inviabiliza essa migração até que todas as funcionalidades da versão antiga do sistema sejam migradas para a nova versão por parte da desenvolvedora do ERP.

- Necessidade de Atualização de Relatórios: Alguns relatórios disponibilizados no sistema ERP para os colaboradores, foram desenvolvidos pela equipe de desenvolvimento da Empresa X na versão antiga do ERP. Com a migração do ERP para a nova versão disponibilizada em SaaS, seria necessário um novo desenvolvimento de grande parte os relatórios disponíveis aos colaboradores.

- Impossibilidade de Parametrizações no Banco de Dados: Para a automatização de determinadas tarefas e rotinas de alguns setores da empresa, alguns gatilhos são configurados no próprio sistema de gerenciamento do banco de dados da empresa. Com a utilização do ERP na modalidade SaaS, todas as parametrizações de banco de dados são removidas pela empresa desenvolvedora do ERP, que nessa modalidade mantém autonomia total sob o banco de dados, não sendo mais possível acesso direto ao banco de dados e consequentemente a parametrização e configuração desses gatilhos. Esse formato aumentaria a carga de trabalho de diversos setores que agora deverão fazer manualmente as rotinas que antes eram automatizadas através dos gatilhos de banco de dados. 
- Integração e Compatibilidade de Sistemas de Terceiros: Para suprir algumas funcionalidades em que o sistema ERP atual não consegue atender de forma satisfatória a Empresa $\mathrm{X}$, alguns sistemas de terceiros são utilizados para determinadas rotinas e controles de diversos setores, estes sistemas de terceiros fazem a integração com o sistema ERP através do banco de dados, sendo possível a geração de relatórios com os dados integrados de todos os setores. Com a migração do banco de dados para o ambiente SaaS e consequentemente a perda da possibilidade de acesso e parametrização do banco de dados, não é possível a integração de sistemas de terceiros com o sistema ERP, inviabilizando a migração na modalidade atual.

- Tempo para Resolução de Ocorrências: Com o sistema ERP implantado em ambiente On-Premises, a resolução de possíveis problemas pela equipe interna tende a se tornar mais rápida. Com o ambiente ERP implantado na modalidade SaaS, o prazo acordado para o atendimento de ocorrências críticas é elevado, o que poderia causar um grande impacto na produção da Empresa $\mathrm{X}$ em caso da indisponibilidade de algum serviço ou funcionalidade.

- Investimento em Infraestrutura de Comunicação: Para garantir a disponibilidade do sistema ERP em níveis aceitáveis para o negócio, é necessário um investimento em links de comunicação redundantes, garantindo a disponibilidade do sistema ERP em caso da falha de um dos links de internet.

Para a realização dos testes de desempenho com a aplicação no ambiente SaaS, foram realizadas três execuções do processo de cálculo do mesmo projeto utilizado nos testes anteriores, os resultados apresentados na Figura 3 foram obtidos em comparação aos resultados anteriores:

Figura 3 - Comparação do Tempo de Cálculo de Projeto entre a Infraestrutura On-Premises e Cloud Computing.

\section{Tempo de Execução do Processo de Cálculo de Projeto (s)}

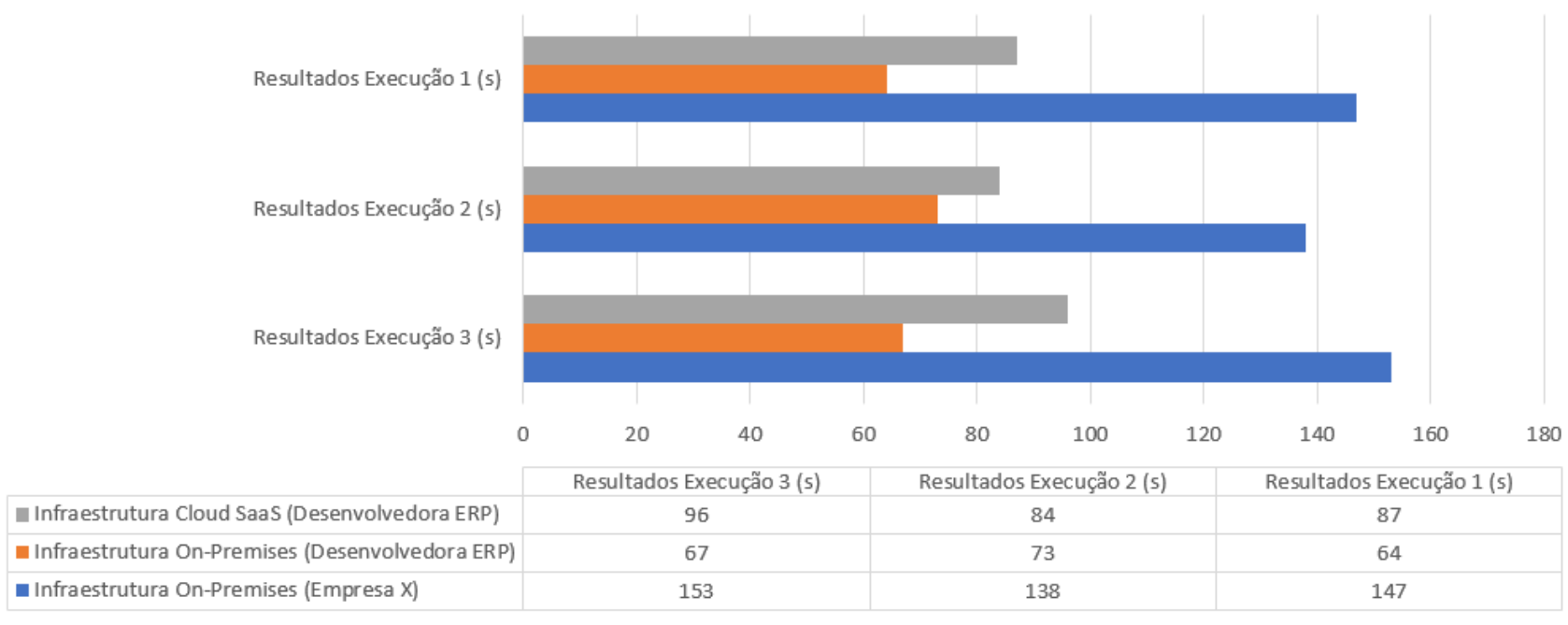

Fonte: Autores (2021).

\section{Resultados}

Com a análise realizada no cenário de atualização do ambiente de servidores On-premises e nos resultados obtidos com a realização da POC com o sistema ERP no ambiente SaaS, a tomada de decisão foi realizada pelo nível estratégico da Empresa X. Após analisar os diversos impactos e problemas que impediriam a utilização imediata do sistema ERP na 
modalidade SaaS, e a falta alinhamento entre o cenário proposto pela empresa desenvolvedora do ERP e os requisitos e regras de negócio da Empresa X, os gestores da Empresa X optaram por manter a infraestrutura de servidores On-premises, realizando a aquisição e atualização dos equipamentos e softwares conforme apresentado no item 3.3.1.

Com a finalização do projeto de aquisição dos novos equipamentos com a tecnologia SSD - atendendo aos requisitos recomendados pela empresa desenvolvedora do ERP - a atualização do sistema de HyperVisor e a atualização da versão do banco de dados, uma grande melhoria de desempenho foi perceptível em todo o ambiente de servidores virtuais. Para a validação do ganho de desempenho obtido com a finalização do projeto, novos testes de desempenho no sistema ERP foram realizados, utilizando os mesmos parâmetros já utilizados nos testes anteriores.

Para os testes no novo ambiente de servidores On-premises, novamente foram realizadas três execuções do processo de cálculo do mesmo projeto utilizado anteriormente. A Figura 4 apresenta os resultados obtidos, em comparação aos resultados anteriores:

Figura 4 - Comparação do Tempo de Cálculo de Projeto Após Melhorias no Ambiente On-Premises.

Tempo de Execução do Processo de Cálculo de Projeto (s)

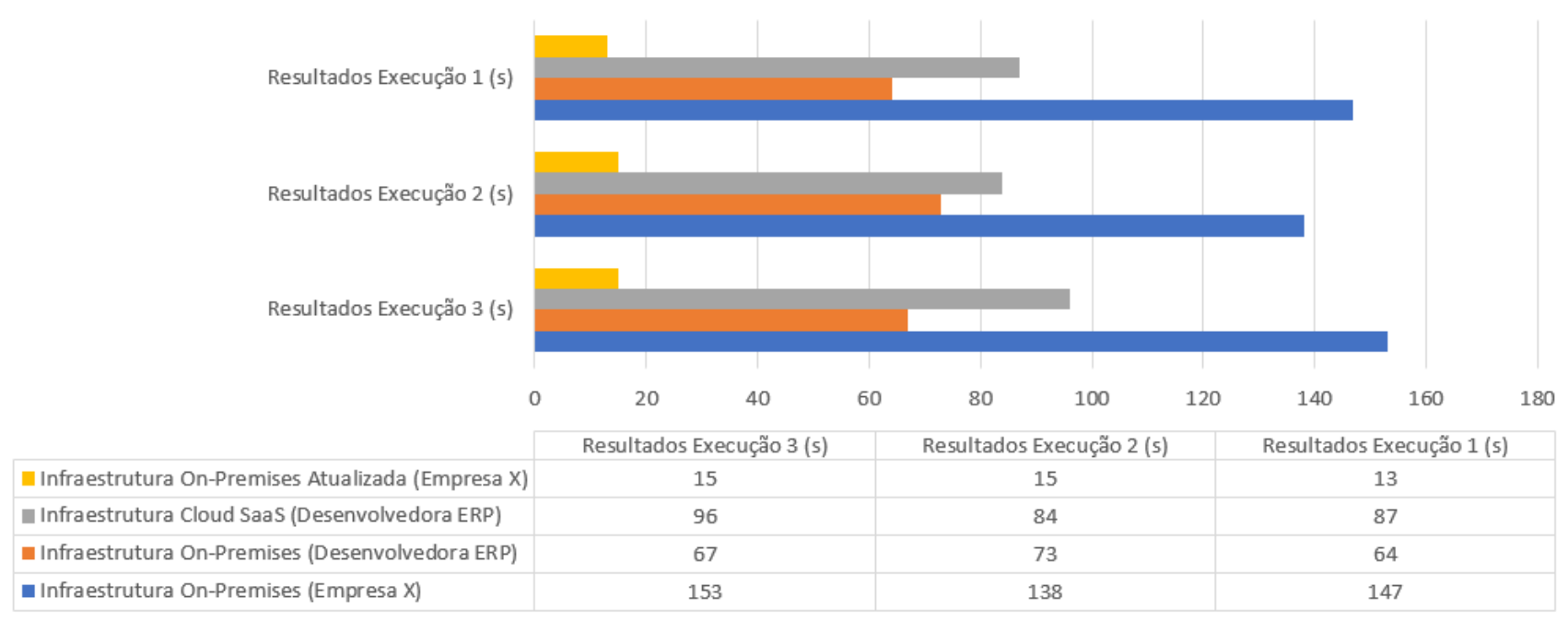

Fonte: Autores (2021).

Com a análise dos resultados obtidos no teste final, pode-se notar uma redução no tempo médio de execução do processo de cálculo de projeto de cerca de $90 \%$, comparando o tempo percorrido do início do processo até sua finalização, utilizando o novo ambiente de servidores On-premises em relação a infraestrutura On-premises antiga. Também pode-se notar uma redução no tempo médio de execução de cerca de 83,9\% comparando o novo ambiente de servidores On-premises e o ambiente com o ERP na modalidade Cloud Computing SaaS. Desta forma, pode-se constatar que o objetivo inicial do projeto foi alcançado e os resultados obtidos foram satisfatórios para o negócio.

\section{Considerações Finais}

Para a Empresa estudada a viabilidade técnica se mostrou mais eficaz a manutenção da infraestrutura de servidores On-premises. Diante disso, comprovou-se que a aquisição e atualização dos equipamentos e softwares é mais vantajosa conforme demonstrado na Figura 4.

Apesar da tecnologia de Cloud Computing ser inovadora e trazer diversos benefícios para as empresas que aderem ao modelo, para a Empresa $\mathrm{X}$ não foi a opção adotada visto que não mostrou ganho de performance tal como demonstrado na Figura 3. 
Também pôde-se constatar que a escolha por um modelo de implementação da tecnologia de Cloud Computing pode refletir diretamente no resultado do projeto. No estudo de caso realizado, o modelo escolhido para a simulação da migração do sistema ERP foi o SaaS. Por se tratar de um modelo onde a organização não tem autonomia para realizar configurações e parametrizações nos servidores, por conter diversas restrições quanto a integração com sistemas de terceiros, e por gerar uma grande demanda da equipe de desenvolvimento interna para ajuste de relatórios e funcionalidades, o modelo SaaS tornou o projeto de migração do ERP On-premises para Cloud inviável para esta organização. A opção por uma metodologia diferente de implementação do ERP em ambiente Cloud Computing, que entregue uma autonomia maior no ambiente de servidores para a Empresa $\mathrm{X}$, em relação a metodologia IaaS, proporcionaria à Empresa $\mathrm{X}$ a possibilidade de realizar as parametrizações necessárias no banco de dados e reduziria o tempo na resolução de ocorrências de baixa e média complexidade, o que tornaria o projeto de migração do ERP para Cloud Computing mais viável.

A realização de uma POC, mostrou-se fundamental para expor os diversos problemas que poderiam ocorrer durante o projeto de migração do ERP, também se mostrou de suma importância para uma tomada de decisão mais assertiva, fazendo com que o resultado do projeto estivesse completamente alinhado com as expectativas do negócio, comprovando o conceito apresentado por Carsten (1989).

No projeto estudado, o investimento na infraestrutura interna de servidores, apesar de gerar um maior investimento inicial por parte da organização - em comparação a migração para SaaS - além de trazer o resultado esperado na melhoria de desempenho dos sistemas, gerou pouco impacto na produção da organização durante sua implementação, mostrando-se a tomada de decisão mais efetiva para o cenário atual desta empresa.

Como sugestão para trabalhos futuros, a realização de uma POC utilizando o modelo de infraestrutura como um serviço em nuvem (IaaS) em comparação a infraestrutura on-premises, agregaria o conteúdo abordado neste trabalho, demonstrando a viabilidade, ou não, da migração de um ERP on-premises para cloud computing utilizando IaaS em uma empresa do mesmo segmento onde o estudo de caso atual foi realizado.

\section{Referências}

Opus Software. (2015). PaaS - O que é PaaS? Como começar a usar? Website da Opus Software. https://www.opus-software.com.br/o-que-e-paas-comocomecar-a-usar.

Augusto, H. (2019). SaaS, PaaS e IaaS - Os serviços de computação em nuvem. Website da QiNetwork. https://www.qinetwork.com.br/saas-paas-iaas-osservicos-de-computacao-em-nuvem/.

Carsten, B. (1989). Carsten's Corner. Power conversion and intelligent motion magazine, 38. Intertec Publishing Corp. 0885-0259.

Sispro. (2020). Cloud Computing: Conheça os principais tipos e suas aplicações. Website da Sispro. https://www.sispro.com.br/blog/principais-tipos-decloud-computing/.

Red Hat. (2021). Cloud Computing: Privada, pública, híbrida ou multicloud? Website da Red Hat. https://www.redhat.com/pt-br/topics/cloudcomputing/public-cloud-vs-private-cloud-and-hybrid-cloud.

Ekman, P., Thilenius, P., Windahl, T. (2014). Extending the ERP system: considering the business relationship portfólio. Business Process Management Journal, 20(3), 480-501. 10.1108/BPMJ-08-2012-0085.

Fabiane, F. (2007). Pesquisa qualitativa, exploratória e fenomenológica: Alguns conceitos básicos. Website da Administradores.com. https://administradores.com.br/artigos/pesquisa-qualitativa-exploratoria-e-fenomenologica-alguns-conceitos-basicos.

Gil, A. (1999). Métodos e técnicas de pesquisa social. Atlas São Paulo.

Godoy, A. (1995). Introdução à pesquisa qualitativa e suas possibilidades. Revista de administração de empresas, $35,57-63$.

Gomes, C. (2020). Estudo do paradigma computação em nuvem. Instituto superior de engenharia de Lisboa. Website do Instituto Técnico de Lisboa. http://hdl.handle.net/10400.21/2375.

Hsu, P. (2013). Commodity or competitive advantage? analysis of the ERP value paradox. Electronic commerce research and applications, 12(6), 412-424. 10.1016/jelerap.2013.06.004

Junior, J., Pires, S. (2010). Sistemas integrados de gestão ERP e cloud computing: características, vantagens e desafios. Anais SIMPOI 2010, p.1 
Research, Society and Development, v. 10, n. 9, e51510918340, 2021

(CC BY 4.0) | ISSN 2525-3409 | DOI: http://dx.doi.org/10.33448/rsd-v10i9.18340

Leite, D. (2020). Platform as a Service: o que é PaaS? Website Blog Vindi. https://blog.vindi.com.br/platform-as-a-service-paas.

Machado, W. (2003). O Estudo de caso como método de pesquisa científica. Website da Classe Contábil. https://classecontabil.com.br/o-estudo-de-casocomo-metodo-de-pesquisa-cientifica.

Beheshti, H., Blaylock, B., Henderson, D., Lollar, J. (2014). Selection and critical success factors in successful ERP implementation. Competitiveness review an international business journal. Journal of global competitiveness 24(4), 357-375. 10.1108/CR-10-2013-0082.

Microsoft Corporation. (2021). Microsoft SQL Server 2008 R2 Lifecycle. Website da Microsoft Corporation. https://docs.microsoft.com/ptbr/lifecycle/products/microsoft-sql-server-2008-r2.

Synnex Westcon-Comstor. (2021). O que é uma rede de armazenamento (SAN) e quais os benefícios. Website da Synnex. https://digital.br.synnex.com/o-queuma-rede-de-armazenamento- san-e-quais-os-beneficios.

Vmware. (2021). O que é um hypervisor. Website da Vmware. https://www.vmware.com/br/topics/glossary/content/hypervisor.html.

Passos, E. (2011). Storage, um item essencial. Website da Infobusiness. https://infob.com.br/storage-um-item-essencial.

SEBRAE. (2021). POC (Proof of Concept): O que é e por que é importante para softwares. Website do SEBRAE. https://inovacaosebraeminas.com.br/pocproof-of-concept.

Gaea. (2021). Por que é importante fazer uma prova de conceito? (POC). Website do Gaea. https://gaea.com.br/por-que-e-importante-fazer-uma-prova-deconceito.

Rosa, D. (2021). Os 6 pilares fundamentais para sua longa $e$ única jornada para Nuvem. Website Jornada para nuvem. http://jornadaparanuvem.com.br/fundamentos-de-cloud-computing/separacao-da-responsabilidade.

Teles, G. (2020). Como não ficar confuso com conceitos de computação em nuvem. Website Guilherme Teles. https://guilhermeteles.com.br/como-nao-ficarconfuso-com-conceitos-de-computacao-em-nuvem.

Trivinõs, A. (1987). Introdução à pesquisa em ciências sociais: a pesquisa qualitativa em educação. Atlas São Paulo. (5a ed.), 175.

Totvs. (2020). O que é ERP? Website da Totvs. https://www.totvs.com/blog/erp/o-que-e-erp 